\title{
Stratified follow-up pathways for cancer patients
}

\author{
Author: Hugo De La Peña*
}

\section{Summary}

> Overstretched/overbooked clinics and patients on waiting lists compromise patient care, safety, experience and go fundamentally against core NHS values.

$>$ A change in the way patients are followed up is therefore urgently needed.

> The new model applies to cancer patients following strict inclusion and exclusion criteria for a total of 5 years when patients are ultimately discharged.

> A personalised remote follow-up (FU) schedule is created for each patient, based on European Association of Urology (EAU) guidelines.

> Wellbeing events and holistic needs assessment are mandatory for patients entering the new FU model.

> Expert information technology (IT), admin, cancer nurse specialist (CNS) and rapid access support is available on demand for patients on the new FU pathway.

> Robust IT and admin systems are in place to avoid never events: losing patients to FU and cancer relapses not picked up in timely fashion.

> Patients will avoid 10 to 13 clinic appointments/hospital visits each under the new model.

> The new model is being piloted in Oxford before expanding it to the wider NHS Network.

\section{Introduction}

Due to previously successful quality improvement (QI) projects within the germ cell cancer team at Oxford University Hospitals $(\mathrm{OUH})$, we simplified surveillance cancer protocols ${ }^{1}$ and developed UK national guidelines. ${ }^{2}$ We therefore decided to stratify cancer follow-up regimes in order to improve both the pathway and patient experience. This model is currently being tested in testicular cancer, but to be implemented in prostate cancer and other tumour sites as well.

\section{Materials and methods}

> The new model includes face-to-face clinic review for the first year post orchidectomy followed by virtual/remote monitoring using IT systems, cancer nurse specialists (CNS) and admin expert support for a total of 5 years based on European (EAU)

Author: Oxford University Hospitals NHS Foundation Trust, UK ${ }^{*} \mathrm{RCP}$ chief registrar guidelines once the first surveillance computed tomography (CT) has been performed, reported as normal and discussed with the patient in clinic.

$>$ A personalised schedule with routine surveillance tumour markers in blood (6-monthly until year 5 for both seminomas and non-seminomas) and CT at 3 and 5 years post orchidectomy (for seminomas) and as per TEO8 and TRISST trials for nonseminomas will be requested and provided to patients.

> Patients need to attend one of the four mandatory wellbeing events running through the year and receive a holistic needs assessment (HNA).

> Eligibility: Stage 1 seminoma or non-seminomatous cancer patients post orchidectomy with or without adjuvant chemotherapy after the first year on standard surveillance with $\mathrm{CT}$ report at year 1 showing no evidence of cancer. Motivated/ engaged patients keen to participate and embrace the new model with Low anxiety scores as measured by routine psychological medicine questionnaires before appointments throughout the last 12 months prior to entering the new pathway.

> Ineligibility: Patients with metastatic disease or on active anticancer treatment. Learning disabilities. In prison or under special needs/care requirements. Patients who have failed to attend standard face-to-face clinic follow-ups in the last 12 months (DNAs: did not attend). High anxiety scores as measured by psychological medicine questionnaires.

\section{Results and discussion}

The EAU guidelines (2015) and Kollmannsberger et al (2014) looked at data from centres across the world and found that most relapses (around 98\%) occur in the first 3 years, with the highest proportion in year one, ${ }^{3,4}$ also confirming that clinical examination does not pick up cancer relapse.

With increasing numbers of patients now living with and beyond cancer due to better diagnosis, cure rates and treatment modalities, there is irrefutable and palpable evidence of increased demand on the service. NHS England is aware that the NHS cannot cope with continuing to treat and follow up patients with a cancer diagnosis in the current way. ${ }^{5}$ Increased demand is thought to be approximately 3\% / year (NHS Improvement 2016) and a need to redistribute resources has been identified and prioritised as a matter of urgency as overstretched clinics compromise patient care, safety and patient experience.

\section{Conclusion}

The new stratified follow up pathway for testicular cancer patients in Oxford is now in place and due to be implemented 
to the wider Thames Valley Supra Regional Network and other tumour sites at $\mathrm{OUH}$. The pathway proposed will enable patients to become experts in their own disease, take ownership of their health and return to as near a normal life as possible sooner after diagnosis without compromising care or safety and improving patient experience at the same time. We anticipate up to $70 \%$ of our follow-up patients ( $n=150-200$ ) to be eligible to enter the pathway in due course. We have created a robust database, which is monitored by dedicated admin, IT and cancer specialist teams in order to ensure patients are never lost to follow up and that relapses in the community are picked up immediately. We do not know in full at this stage the financial implications this change will bring, tariffs to the new service will also apply, penalties for waiting lists will be avoided as well as paying locums to do extra clinics. Regardless and above costs, we accept that overstretched clinics compromise patient care, safety and experience and therefore change is urgently needed according to our NHS core values.

\section{References}

1 De La Peña $\mathrm{H}$. No longer any role for routine follow-up chest $X$-rays in men with stage I germ cell cancer. Eur ] Cancer 2017;84:354-9.

2 Watson RA and De La Peña H. Development of a best-practice clinical guideline for the use of bleomycin in the treatment of germ cell tumours in the UK. $\mathrm{Br}$ ] Cancer 2018;119:1044-51.

3 Oldenburg J. Testicular seminoma and non-seminoma: ESMO Clinical Practice Guidelines for diagnosis, treatment and follow-up. Ann Oncol 2013;24(Suppl 6):vi125-32.

4 Kollmannsberger $C$. Patterns of relapse in patients with clinical stage I testicular cancer managed with active surveillance. J Clin Oncol 2015;33:51-7.

5 NHS England. Achieving world-class cancer outcomes: Taking the strategy forward. NHS England, 2016. 